\title{
Magnetic study of an amorphous conducting polyaniline
}

\author{
Debangshu Chaudhuri, Ashwani Kumar, and D. D. Sarma* \\ Solid State and Structural Chemistry Unit, Indian Institute of Science, Bangalore-560012, India \\ M. García-Hernández \\ Instituto de Ciencia de Materiales de Madrid, CSIC, Cantoblanco, E-28049 Madrid, Spain \\ Janhavi P. Joshi and S. V. Bhat \\ Department of Physics, Indian Institute of Science, Bangalore 560 012, India
}

\begin{abstract}
We show that newly found $\mathrm{BF}_{3}$-doped polyaniline, although highly conducting, remains amorphous. Magnetic studies reveal many unusual properties, while suggesting that the intrinsic conductivity of this system is significantly larger than all other known forms of conducting polyaniline, establishing it as an interesting class of highly conducting amorphous polymer.
\end{abstract}

Over a number of years, proton-doped polyaniline (PANI) has become one of the most extensively studied conducting polymers owing to its high electrical conductivity and consequent possibilities of technological applications. Undoped polyaniline is amorphous. However, proton doping induces the formation of highly conducting, crystalline domains separated by amorphous, insulating barriers. ${ }^{1}$ It is reported that the doped charge carriers in the protonated PANI are predominantly localized in the amorphous regions, giving rise to local moments, while a relatively small fraction of the doped carriers has three-dimensionally extended wave functions in the crystalline parts of the sample. ${ }^{2}$ Since, in the doped PANI the charge and the spins are associated with the same carrier, ${ }^{3}$ magnetic susceptibility and electron spin resonance (ESR) measurements can provide detailed understanding about the microscopic nature of these carriers. We report here a detailed study of the nature of charge carriers in a recently reported conducting PANI, ${ }^{4}$ doped with $\mathrm{BF}_{3}$, which has so far been characterized only by spectroscopic methods. Magnetic susceptibility and temperature dependence of ESR linewidth in conjunction with x-ray diffraction (XRD) results suggest interesting distinctive features of this compound, including an order of magnitude increase in the intrinsic conductivity compared to all other known conducting PANI systems.

The sample was prepared ${ }^{4}$ by doping $\mathrm{BF}_{3}$ into the emeraldine base, synthesized by chemical oxidation of aniline using $\left(\mathrm{NH}_{4}\right)_{2} \mathrm{~S}_{2} \mathrm{O}_{8}$ at $0-5{ }^{\circ} \mathrm{C} .{ }^{5}$ Magnetic susceptibility measurement was carried out with a powdered sample. Since adsorbed molecular oxygen induces ESR line broadening, the powder sample was sealed in a quartz tube under He for those experiments.

In Fig. 1, we show the XRD patterns from undoped PANI and PANI doped with $\mathrm{BF}_{3}$ in comparison to that from HCl-doped PANI, adapted from Ref. 6. The XRD of PANI clearly suggests that the system is amorphous, in agreement with the previous result. ${ }^{6}$ The appearance of a large number

*Also at Jawaharlal Nehru Center for Advanced Scientific Research, Bangalore, India; electronic mail: sarma@sscu.iisc.ernet.in of sharp diffraction peaks upon doping with $\mathrm{HCl}$, suggests significant crystallization of PANI on protonation. In contrast, $\mathrm{BF}_{3}$-doped PANI exhibits an almost identical XRD pattern as the undoped, amorphous PANI, except for the emergence of a weak intensity peak at $2 \theta \approx 24.8^{\circ}$. We find that the position of this peak matches with the most intense peak in the XRD of HCl-PANI. More significantly, we find that the intensity of this peak grows rapidly with progressive time of exposure of the sample to atmosphere during the recording of XRD, as shown in the inset of Fig. 1 in the form of repeated XRD scans of the relevant region. In view of the extreme sensitivity of $\mathrm{BF}_{3}$ to moisture, forming $\mathrm{HF}$ upon hydrolysis, this extraneous peak in $\mathrm{XRD}$ of $\mathrm{BF}_{3}$-doped PANI is easily understood in terms of hydrolyzed $\mathrm{BF}_{3}$ reacting with PANI and giving rise to small crystalline domains of protonated PANI. By minimizing the time of exposure during the measurement, the intensity of this peak could be kept at a

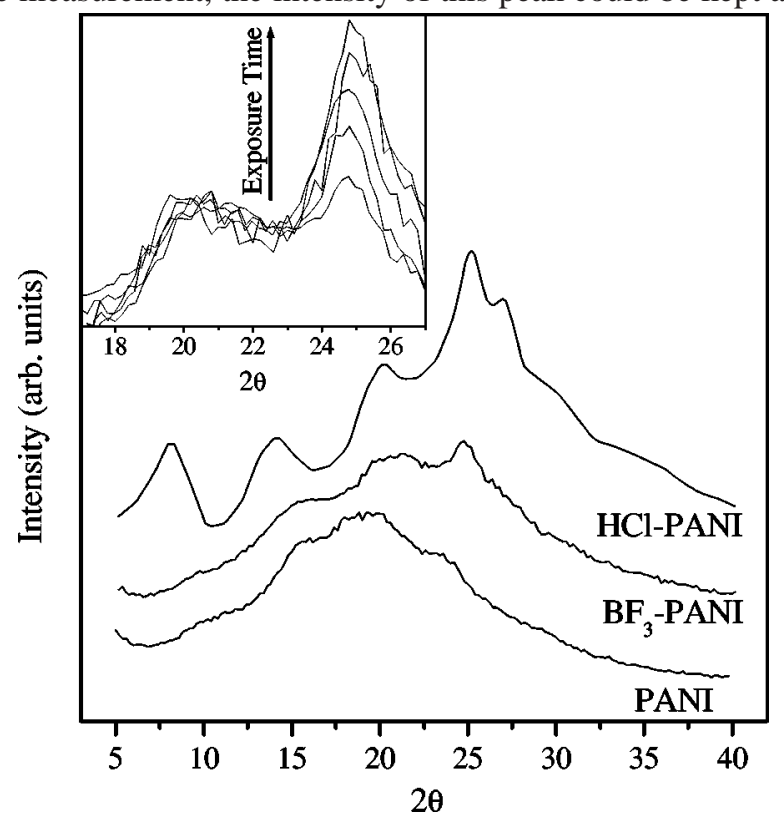

FIG. 1. The XRD patterns of undoped, $\mathrm{BF}_{3}$-doped, and $50 \% \mathrm{HCl}$-doped PANI (reproduced from Ref. 6). Inset shows the progressive gain in the intensity of $24.8^{\circ}$ peak upon exposure to atmosphere. The exposure time in the inset corresponds to 0 and $16 \mathrm{~h}$ for the lowest and the highest patterns, respectively. 

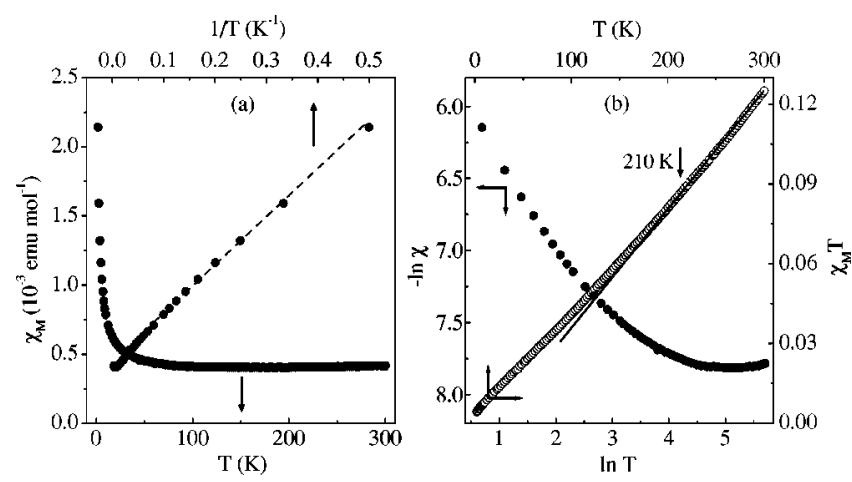

FIG. 2. (a) Variation of dc magnetic susceptibility with temperature and inverse temperature. The dashed line is the fit obtained using the equation $\chi=\chi_{P}+C / T$. (b) Plots of $\chi T$ vs $T$. The solid line is a guide to the eye. $\ln \chi$ vs $\ln T$ suggesting spin delocalization near $210 \mathrm{~K}$.

low level, although it could never be eliminated completely. It is important to note that the conductivity of the sample was found to decrease with increasing exposure to the atmospheric conditions, concomitant with the increase in the intensity of the spurious peak at $24.8^{\circ}$. These observations clearly suggest that in sharp contrast to proton-doped PANI, $\mathrm{BF}_{3}$-doped samples remain amorphous as the undoped PANI. Considering that the intrinsic conductivities of $\mathrm{BF}_{3}$-doped samples are orders of magnitude higher than that of the protonated samples, as discussed later, this observation puts the present system in a unique class of amorphous and yet highly conducting polymers.

We show the susceptibility as a function of temperature in Fig. 2. The experimentally obtained susceptibility value has been normalized to a two-ring repeat unit and finally corrected for the diamagnetic contributions of the atomic cores $\left(\chi_{\text {core }}\right)$ using Pascal constants. ${ }^{7}$ The calculated $\chi_{\text {core }}$ value for $\mathrm{BF}_{3}$ is $193.6 \times 10^{-6} \mathrm{emu} \mathrm{mol}^{-1}$. The susceptibility of doped polyaniline has been described ${ }^{8}$ by $\chi=\chi_{P}+C / T$, where, $\chi_{P}$ is the temperature independent Pauli paramagnetic contribution from the delocalized charge carriers and $C$ is the Curie constant arising from the trapped or localized spins in the polymer. $\chi_{P}$ can in turn be expressed in terms of the average density of states at the Fermi level, $N\left(E_{F}\right)$, by the relation $\chi_{P}=\mu_{B}^{2} N\left(E_{F}\right)$, where $\mu_{B}$ is the Bohr magneton. Thus, $\chi_{P}$ can provide a good estimate of the intrinsic metallic conductivity of the sample, which the bulk resistivity measurement often fails to project, since it depends strongly on the intergrain connectivity in the sample. For instance, the $N\left(E_{F}\right)$ value for camphor-sulfonic acid (CSA) doped PANI, ${ }^{9}$ calculated from the $\chi_{P}$ value is 0.7 states $\mathrm{eV}^{-1}$ (2 rings) ${ }^{-1}$, while that for the $\mathrm{HCl}$-doped sample is 1.6 states $\mathrm{eV}^{-1}$ (2 rings) ${ }^{-1} \cdot{ }^{10}$ This is consistent with the fact that the roomtemperature conductivity of CSA-doped PANI in the pressed-pellet form is $0.047 \mathrm{~S} \mathrm{~cm}^{-1},{ }^{11}$ which is lower than that of powdered HCl-PANI, though PANI doped with CSA has the highest bulk conductivity among the family of doped polyanilines, in thin-film form. ${ }^{12}$

Figure 2(a) shows the susceptibility as a function of temperature ( $\chi$ versus $T$ plot), indicating a nearly temperatureindependent Pauli-like susceptibility in the high-temperature region. The rapid increase of susceptibility with decreasing $T$ follows a typical Curie behavior, as demonstrated by the $\chi$ versus $1 / T$ plot in the same figure. This plot shows the ex- pected linear behavior in the low-temperature regime. The values of $C, \chi_{P}$, and $N\left(E_{F}\right)$ estimated from the best fit of the susceptibility data are $3.7 \times 10^{-3} \mathrm{emu} \mathrm{K} \mathrm{mol}^{-1}, 380$

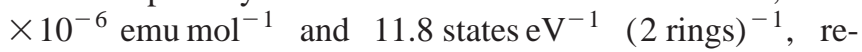
spectively. Thus, the calculated density of states at the Fermi level is considerably higher than that for any other conducting polyaniline system known, typical values reported ${ }^{2}$ so far for various protonated samples being in the range of 1.6 states $\mathrm{eV}^{-1}$ (2 rings) ${ }^{-1}$. Such a higher concentration of delocalized charge carriers in $\mathrm{BF}_{3}$-doped PANI suggests a very high intrinsic conductivity in this system. Also, a high $\chi_{P}$ value in predominantly amorphous $\mathrm{BF}_{3}$-doped PANI is in sharp contrast with the previous results on $\mathrm{HCl}$-doped PANI, ${ }^{6}$ which show that the delocalized carriers and, therefore, $\chi_{P}$, are mainly associated with the crystalline domains in doped PANI. It is possible that the absence of any counterions in $\mathrm{BF}_{3}$-PANI, as opposed to the protonated PANI, may be responsible for these qualitative changes.

Although we have analyzed the magnetic data in terms of $\chi=\chi_{P}+C / T$, close inspection of the temperature dependence suggests interesting deviations from this behavior at $\sim 200 \mathrm{~K}$. For systems obeying this equation, $\chi T$ is linear in temperature. However, $\chi T$ for the $\mathrm{BF}_{3}$-doped PANI shown in Fig. 2(b) exhibits an increase in the slope above $210 \mathrm{~K}$, indicating a slight increase in $\chi_{p}$ and a simultaneous decrease in $C$. While this increase in Pauli susceptibility component is not evident in the $\chi$ versus $T$ plot shown in Fig. 2(a), $\ln \chi$ versus $\ln T$ plot in Fig. 2(b) accentuates a small but gradual increase in the free carrier susceptibility above about $200 \mathrm{~K}$. This enhancement of $\chi_{P}$ at the cost of $C$ with increasing $T$ suggests that these changes are possibly a consequence of the thermal activation of the localized spins into free carriers, indicating that the trapping potential is in the scale of the thermal energy in this system.

We find that the ESR signals from $\mathrm{BF}_{3}$-doped PANI have homogeneously broadened Lorentzian shape, which suggests a three-dimensional delocalization of the charge carriers ${ }^{13}$ as well as a negligible contribution from the hyperfine and dipolar interactions. ${ }^{14}$ The ESR linewidth of a sample is determined by various relaxation processes that influence the lifetime of the spin carriers. We present the temperature dependence of the peak-to-peak linewidth of the ESR signal $\left(\Delta H_{\mathrm{pp}}\right)$ for $\mathrm{BF}_{3}$-PANI in the $4-300 \mathrm{~K}$ range in Fig. 3. Although the lineshape remained essentially Lorentzian in the entire temperature range, the linewidth shows different variations in three different temperature regimes, marked in the figure as A, B, and C. In systems where the scattering between the localized and the delocalized spins determines the relaxation rates, the relaxation time and therefore the linewidth are given by the Korringa relation, ${ }^{15}$ $\Delta H_{\mathrm{pp}} \propto N^{2}\left(E_{F}\right) T$. Between 50 and $230 \mathrm{~K}$ (region B), we indeed find the linewidth to increase linearly with $T$. Since the slope is directly proportional to $N^{2}\left(E_{F}\right)$, we compare the slope in our sample with that of a previously published result on $\mathrm{HCl}$-doped PANI films, ${ }^{14}$ for which the $N\left(E_{F}\right)$ is known to be 1.6 states $\mathrm{eV}^{-1}(2 \text { rings })^{-1}$. From the ratio of the slopes, the calculated $N\left(E_{F}\right)$ for $\mathrm{BF}_{3}$-PANI is 15.2 states $\mathrm{eV}^{-1}(2 \text { rings })^{-1}$; this value matches well with that obtained from the susceptibility results, once again establishing an order of magnitude improvement in the intrinsic conductivity. At further lower temperatures (region A), 


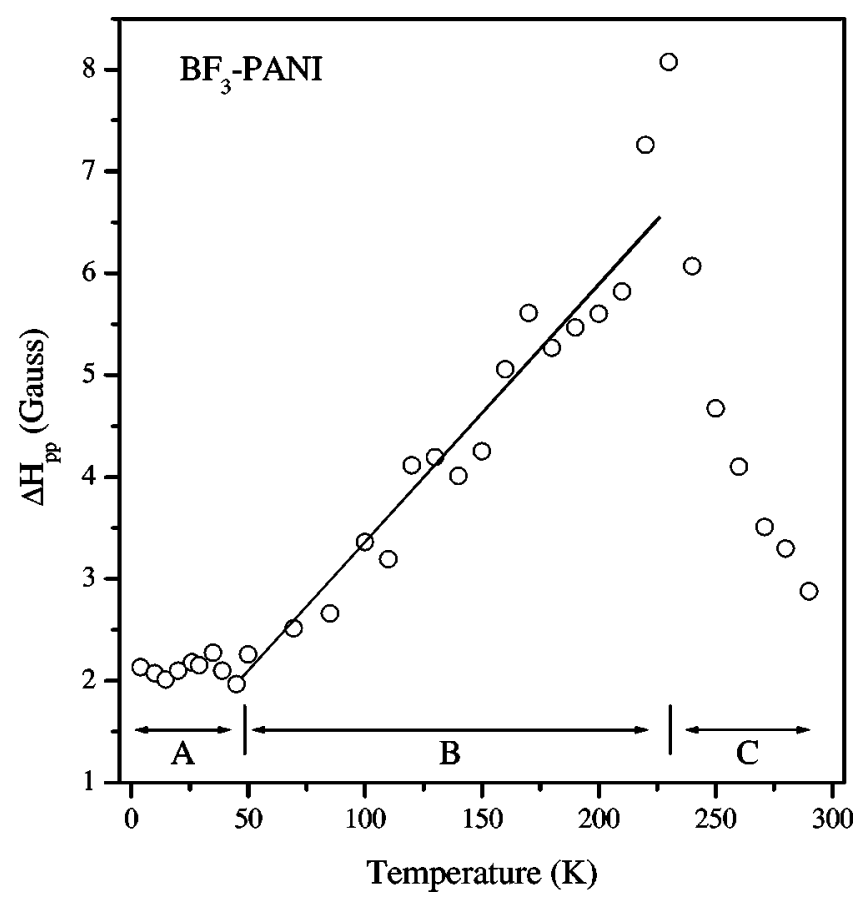

FIG. 3. Plot of peak-to-peak linewidth of the ESR signal vs $T$ for $\mathrm{BF}_{3}$-doped PANI. The solid line indicates the linear $T$ dependence in the intermediate regime.

the linewidth is essentially temperature independent. This is possibly due to a partial localization of the mobile spins, thereby increasing the concentration of fixed spins and compensating the thermally induced changes in the ESR linewidth. A more pronounced effect has been observed ${ }^{14,16}$ in the case of powdered $\mathrm{HCl}$-doped PANI, where the ESR linewidth actually begins to increase with decreasing temperature below $190 \mathrm{~K}$. In more ordered samples like stretched films of HCl-doped PANI, the ESR linewidth at lower temperatures becomes temperature independent, ${ }^{16}$ as observed here. It is therefore quite intriguing to note that despite being amorphous, the effect of localization is so weak in the case of $\mathrm{BF}_{3}$-PANI. It would appear that the energy difference between the chemical potential and the mobility edge in $\mathrm{BF}_{3}$-doped PANI is very small, although the system is extremely disordered. At higher temperatures (region C), we observe a sharp decrease in the ESR linewidth. Since the localized spins are primarily responsible for the relaxation process in ESR, this observation suggests that the number of localized spins decreases at these higher temperatures. This is consistent with the conclusions based on the magnetic sus- ceptibility data [Fig. 2(b)] that the delocalized spins increasingly become mobile above $\sim 210 \mathrm{~K}$, leading to an increase of the $\chi_{P}$ component at the expense of the $C$ value.

In summary, we report an interesting class of conducting polyaniline doped with electron-deficient boron trifluoride. The intrinsic conductivity is at least an order of magnitude higher compared to all other polyaniline samples. This is remarkable in view of the fact that the polymer remains amorphous even on doping, representing a highly conducting amorphous state, at least above $\sim 50 \mathrm{~K}$. This is in sharp contrast to proton-doped PANI, where the conductivity is associated with the crystalline domains. The energy scales associated with the trap potential for localizing spins was found to be small, in order of the thermal energy scale. These distinctive behaviors suggest that the present sample belongs to an interesting class of amorphous and yet highly conducting polymers.

We thank the Council for Scientific and Industrial Research (CSIR), Government of India, for financial support.

${ }^{1}$ A. J. Epstein, J. M. Ginder, F. Zuo, R. W. Bigelow, H. S. Woo, D. B. Tanner, A. F. Richter, W. S. Huang, and A. G. MacDiarmid, Synth. Met. 18, 303 (1987).

${ }^{2}$ Z. H. Wang, C. Li, E. M. Scherr, A. G. MacDiarmid, and A. J. Epstein, Phys. Rev. Lett. 66, 1745 (1991).

${ }^{3}$ K. Mizoguchi, M. Nechtschein, J. P. Travers, and C. Menardo, Phys. Rev. Lett. 63, 66 (1989).

${ }^{4}$ D. Chaudhuri, A. Kumar, I. Rudra, and D. D. Sarma, Adv. Mater. 13, 1548 (2001).

${ }^{5}$ A. G. MacDiarmid, J. C. Chiang, A. F. Richter, and A. J. Epstein, Synth. Met. 18, 285 (1987).

${ }^{6}$ M. E. Jozefowicz, R. Laversanne, H. H. S. Javadi, A. J. Epstein, J. P. Pouget, X. Tang, and A. G. MacDiarmid, Phys. Rev. B 39, 12958 (1989).

${ }^{7}$ J. H. Van Vleck, The Theory of Electric and Magnetic Susceptibilities (Oxford University Press, New York, 1966).

${ }^{8}$ J. M. Ginder, A. F. Richter, A. G. MacDiarmid, and A. J. Epstein, Solid State Commun. 63, 97 (1987).

${ }^{9}$ Y. Cao and A. J. Heeger, Synth. Met. 52, 193 (1992).

${ }^{10}$ N. S. Sariciftci, A. J. Heeger, and Y. Cao, Phys. Rev. B 49, 5988 (1994).

${ }^{11}$ J. Stejskal, I. Sapurina, M. Trchova, J. Prokes, I. Krivka, and E. Tobolkova, Macromolecules 31, 2218 (1998).

${ }^{12}$ M. Reghu, Y. Cao, D. Moses, and A. J. Heeger, Phys. Rev. B 47, 1758 (1993).

${ }^{13}$ J. Joo, V. N. Prigodin, Y. G. Min, A. G. MacDiarmid, and A. J. Epstein, Phys. Rev. B 50, 12226 (1994).

${ }^{14}$ Z. H. Wang, E. M. Scherr, A. G. MacDiarmid, and A. J. Epstein, Phys. Rev. B 45, 4190 (1992).

${ }^{15}$ S. E. Barnes, Adv. Phys. 30, 801 (1981); J. Korringa, Physica 16, 601 (1950).

${ }^{16}$ J. Joo, S. M. Long, J. P. Pouget, E. J. Oh, A. G. MacDiarmid, and A. J. Epstein, Phys. Rev. B 57, 9567 (1998). 